\title{
Genome assembly and annotation of Nothobranchius rachovii killifish
}

\author{
Zulfiia Guvatova \\ Center for Precision Genome Editing \\ and Genetic Technologies for \\ Biomedicine \\ Engelhardt Institute of Molecular \\ Biology, Russian Academy of Sciences \\ Moscow, Russia \\ guvatova.zulfiya@mail.ru \\ Artemy Tokarev \\ Veterinary biology faculty \\ MSAVM\&B - MVA named after K.I. \\ Skryabin \\ Moscow, Russia \\ artemiy.tokarev@mail.ru
}

\author{
George Krasnov \\ Center for Precision Genome Editing \\ and Genetic Technologies for \\ Biomedicine \\ Engelhardt Institute of Molecular \\ Biology, Russian Academy of Sciences \\ Moscow, Russia \\ gskrasnov@mail.ru \\ Maria Fedorova \\ Laboratory of Postgenomic Research \\ Engelhardt Institute of Molecular \\ Biology, Russian Academy of Sciences \\ Moscow, Russia \\ fedorowams@yandex.ru
}

\author{
Anastasiya Snezhkina \\ Center for Precision Genome Editing \\ and Genetic Technologies for \\ Biomedicine \\ Engelhardt Institute of Molecular \\ Biology, Russian Academy of Sciences \\ Moscow, Russia \\ leftger@rambler.ru \\ Anna Kudryavtseva \\ Center for Precision Genome Editing \\ and Genetic Technologies for \\ Biomedicine \\ Engelhardt Institute of Molecular \\ Biology, Russian Academy of Sciences \\ Moscow, Russia \\ rhizamoeba@mail.ru
}

\begin{abstract}
Nothobranchius rachovii is a species of freshwater annual killifish native to Africa. This organism is an attractive model in aging studies because of short lifetime and rapid maturation. In this study, we present draft genome assembly of this organism based on Nanopore reads and polished with Illumina reads. We tested four popular Nanopore reads assemblers (Flye, MaSuRCA, Shasta, wtdbg2) and two polishers (Pilon and MaSuRCA-polish tool). We selected the best combination of these tools in terms of contig length and assembly completeness.
\end{abstract}

Keywords: Nothobranchius rachovii, genome assembly, Nanopore,

\section{Introduction}

Nothobranchius rachovii is a species of killifish native to Africa (southern Mozambique). Due to very short duration of the rain season, the natural lifespan of these animals is limited to a few months and their captive lifespan is likewise short. Along with $N$. furzerii, another species from this genus, $N$. rachovii is an attractive model system for ageing and disease research $[1,2]$. With respect to the molecular, cellular, and integrative traits of aging, these species show significant similarities to mammals, including humans [1, 2]. The complete genome assembly of $N$. rachovii still lacks.

The genome of $N$. furzerii, a close phylogenetic relative of $N$. rachovii [3], is abnormally enriched with tandem repeats ( $21 \%$ of its length), which has been suggested as a factor in its fast ageing [4]. On other hand, this complicates the genome assembly and make challenging to get high accuracy, attractive contig lengths stats and low misassembly rate.

In recent years, Oxford Nanopore Technology (ONT) sequencers are widely used to produce high-quality and inexpensive genome assemblies of various organisms, including those with complex genomic structure (low complexity, abundance of tandem repeats, pronounced heterozygosity, polyploidy). The field of the development of software for the genome assembly based on ONT reads is one of the fastest growing at the moment.

In this study, we aimed to derive high accuracy genome assembly of $N$. rachovii based on ONT and Illumina reads. For this purpose, we tested several most popular and recent assemblers and polishers, compared the results in terms of assembly completeness, contig lengths and similarity to the assembled genome of $N$. furzerii.

\section{Materials and Methods}

\section{Sample preparation, sequencing}

Genomic DNA was extracted from muscle tissue of N. rachovii using DNeasy Blood and Tissue Kit (QIAGEN, USA) according to the manufacturers instructions. To generate the long reads DNA library from $1 \mu \mathrm{g}$ of high molecular weight DNA was performed following the SQK LSK108 protocol (Oxford Nanopore Technologies, UK). Sequencing was performed on five R9.4.1 flow cells on a MinION (Oxford Nanopore Technologies, UK). The shortreads was generated using HiSeq 2000 Sequencing System (Illumina, USA) according to manufacturer's instructions. The libraries for sequencing on HiSeq 2000 System (Illumina, USA) were constructed using $500 \mathrm{ng}$ of genomic DNA using the TruSeq DNA LT kit(Illumina, USA) according to the manufacturer's instructions. RNA extracted from muscle tissue of fish was used for library construction and RNA sequencing (RNA-seq) on NextSeq500 System (Illumina, USA).

\section{Genome assembly and annotation}

First, we derived nucleotide sequences (fastq) from the obtained raw electrical signal intensity data (fast5) using Guppy and fast "flip-flop" high accuracy calling algorithm. Using trimmomatic we filtered out low-quality reads with average phred-scaled Q $<4$ (no trimming has been applied). Next, we performed primary genome assemblies of $N$. rachovii genome (based on ONT reads) using four different assemblers: Flye (2.7), MaSuRCA (3.3.4), Shasta (0.4.0), and wtdbg2 (2.5). The derived contigs were polished by Racon (1.4.3) with ONT reads (2 iterations) and then polished with Illumina reads parallelly by Pilon (1.23) and by MaSuRCApolish tool (3.3.4).

At this step, we calculated assembly metrics using QUAST and evaluated assembly completeness using BUSCO (Vertebrata and Actinopterygii datasets). For the further 
analysis, we selected only the best assembly. Additionally, we measured the efficacy of polishers, also by BUSCO metrics.

Next, we excluded redundant contigs (coming from diploidy or mis-assembled contigs) using PurgeHaplotigs tool (Feb 2020). At this step, we adjusted PurgeHaplotigs parameters in order to maximally shorten assembly length, but not to impair its completeness (in terms of BUSCO metrics). Finally, the derived pool of contigs was mapped to the database of bacterial genomes (MiniKraken DB) in order to eliminate contaminants.

The derived contigs were mapped to the reference genome of Nothobranchius furzerii (CCSH00000000.1) and indel/SNP rates were evaluated. Dotplots were generated by LAST and mummer. Additionally, we tried to find orthologous between $N$. furzerii and $N$. rachovii genes using OrthoFinder.

The annotation of the assembled contigs was performed by funannotate (1.7.0) pipeline. For this purpose, we performed transcriptome assembly of $N$. rachovii RNA-Seq reads using Trinity (genome-guided), and then the derived transcripts were mapped to the genomic contigs using minimap2. These BAM files were transferred to the PASA pipeline (incorporated into funannotate) in order to train Augustus, snap, GlimmerHMM and pass the derived results to the EvidenceModeler. After filtering gene models, gene annotation was performed (using UniProt, BUSCO, eggNOG, InterProScan databases).

\section{Results}

In the present work, we performed assembly of N. rachovii genome using ONT reads and four assemblers. Among them, Flye demonstrated the best results in terms of contig lengths and assembly completeness. After eliminating redundant contigs, the genome length comprised $1183 \mathrm{Mb}$ with N50 = $7.5 \mathrm{Mb}$. The completeness of the assembly was evaluated as 98.6\% (completed/duplicated BUSCOs; Vertebrata dataset), 95.4\% (Actinopterygii dataset). Shasta gave worse results in terms of assembly completeness, whereas MaSuRCA lower N50 with comparable assembly completeness. wtdbg2 demonstrated the worse result.
26.897 protein-coding genes have been identified in the assembly. There were about 1.8 alternatively spliced transcript variants per gene. Besides, we identified 231 tRNA genes. The length of mitochondrial genome comprised $21 \mathrm{~Kb}$, contained 23 tRNAs and 13 protein-coding genes. For 19.844 genes we did find orthologs in $N$. furzerii reference genome. The overall sequence similarity between $N$. furzerii and $N$. rachovii comprised $82.5 \%$ for the entire genome, $93.5 \%$ for coding nucleotide sequences, and $96 \%$ for the encoded protein sequences.

\section{Consclusions}

In this study, we present a draft genome assembly of Nothobranchius rachovii, a species of killifish native to Africa. This organism is considered at attractive model system for ageing and disease research. Its genome shares high homology to the Nothobranchius furzerii and is highly enriched with tandem repeats, which is thought to be one of the reasons for its rapid aging.

\section{ACKNOWLEDGMENT}

This work was supported by grant 075-15-2019-1660 from the Ministry of Science and Higher Education of the Russian Federation.

\section{REFERENCES}

[1] C.Y. Hsu, Y.C. Chiu, W.L. Hsu, Y.P. Chan. "Age-related markers assayed at different developmental stages of the annual fish Nothobranchius rachovii," J. Gerontol. A. Biol. Sci. Med. Sci., vol. 63(12), pp.1267-76, Dec 2008. doi: 10.1093/gerona/63.12.1267

[2] M. Platzer, C. Englert. "Nothobranchius furzeri: A Model for Aging Research and More," Trends. Genet., vol. 32 (9), pp. 543-552, Sept 2016. doi: 10.1016/j.tig.2016.06.006.

[3] A. Dorn, Musilová Z, Platzer M, Reichwald K, Cellerino A. "The strange case of East African annual fishes: aridification correlates with diversification for a savannah aquatic group?" BMC Evol Biol, vol. 14, p.210, 2014 Oct. doi: 10.1186/s12862-014-0210-3.

[4] K. Reichwald, C. Lauber, et al. "High tandem repeat content in the genome of the short-lived annual fish Nothobranchius furzeri: a new vertebrate model for aging research," Genome Biol, vol. 10(2), R16, Feb 2009. doi: 10.1186/gb-2009-10-2-r16. 